\title{
Topological sensitivity analysis in heterogeneous anisotropic elasticity problem. Theoretical and Computational Aspects.
}

\author{
S.M. Giusti ${ }^{\mathrm{a}, *}$, A. Ferrer ${ }^{\mathrm{b}, \mathrm{c}}$, J. Oliver ${ }^{\mathrm{b}, \mathrm{d}}$ \\ ${ }^{a}$ National Technological University, Regional Faculty of Córdoba (UTN/FRC - CONICET), Maestro M. López esq. Cruz Roja Argentina, \\ X5016ZAA - Córdoba, Argentina. \\ ${ }^{b}$ CIMNE - Centre Internacional de Metodes Numerics en Enginyeria, Campus Nord UPC, Edifici C-1, c/Jordi Girona 1-3, 08034 Barcelona, \\ Spain. \\ ${ }^{c}$ Escola Superior d'Enginyeries Industrial, Aeroespacial i Audiovisual de Terrassa, Campus de Terrassa, Edificio TR45. C. Colom, 1108222 \\ Terrassa, Spain \\ ${ }^{d}$ E.T.S. d'Enginyers de Camins, Canals i Ports, Technical University of Catalonia, Campus Nord UPC, Edifici C-1, c/Jordi Girona 1-3, 08034 \\ Barcelona, Spain.
}

\begin{abstract}
The topological sensitivity analysis for the heterogeneous and anisotropic elasticity problem in two-dimensions is performed in this work. The main result of the paper is an analytical closed-form of the topological derivative for the total potential energy of the problem. This derivative displays the sensitivity of the cost functional (the energy in this case) when a small singular perturbation is introduced in an arbitrary point of the domain. In this case, we consider a small disc with a completely different elastic material. Full mathematical justification for the derived formula, and derivations of precise estimates for the remainders of the topological asymptotic expansion are provided. Finally, the influence of the heterogeneity and anisotropy is shown through some numerical examples of structural topology optimization.
\end{abstract}

Keywords: topological derivative, structural optimization, topology design, elastic anisotropy

\section{Introduction}

Topological asymptotic analysis allows obtaining an asymptotic expansion of a given shape functional when a geometrical domain is singularly perturbed. This perturbation can be materialized by the insertion of holes, inclusions, source-terms or even cracks. The main concept arising from this analysis is the topological derivative [1,2]. This derivative measures the sensitivity of the shape functional with respect to the infinitesimal singular domain perturbation and it was rigorously introduced in [3]. Since then, this concept has proven extremely useful in the treatment of a wide range of problems; see, for instance, $[4,5,6,7,8,9,10,11]$. Concerning the theoretical development of the topological asymptotic analysis, besides the monograph [1], the reader is referred to [12, 13, 14, 15].

In order to introduce these concepts, let us consider an open and bounded domain $\Omega \subset \mathbb{R}^{2}$, see figure 1 , which is subject to a non-smooth perturbation confined in a small region $\omega_{\varepsilon}(\widehat{x})=\widehat{x}+\varepsilon \omega$ of size $\varepsilon$. Here, $\widehat{x}$ is an arbitrary point of $\Omega$ and $\omega$ is a fixed domain of $\mathbb{R}^{2}$. Then, we assume that a given shape functional $\mathcal{J}_{\varepsilon}(\Omega)$, associated to the topologically perturbed domain, admits the following topological asymptotic expansion [1]

$$
\mathcal{J}_{\varepsilon}(\Omega)=\mathcal{J}(\Omega)+f(\varepsilon) \mathcal{T}(\widehat{x})+o(f(\varepsilon)),
$$

where $\mathcal{J}(\Omega)$ is the shape functional associated to the unperturbed domain and $f(\varepsilon)$ is a positive function such that $f(\varepsilon) \rightarrow 0$ when $\varepsilon \rightarrow 0^{+}$. The function $\widehat{x} \mapsto \mathcal{T}(\widehat{x})$ is termed the topological derivative of $\mathcal{J}$ at $\widehat{x}$. Therefore, the term $f(\varepsilon) \mathcal{T}(\widehat{x})$ represents a first order correction of $\mathcal{J}(\Omega)$ to approximate $\mathcal{J}_{\varepsilon}(\Omega)$ in $\widehat{x}$. In this work, the singular perturbation is characterised by a circular disc, denoted $B_{\varepsilon}$, with boundary $\partial B_{\varepsilon}$ and different constitutive properties, see figure 1 .

\footnotetext{
${ }^{*}$ Corresponding author Oliver)

Email addresses: sgiusti@frc.utn.edu.ar (S.M. Giusti), aferrer@cimne.upc.edu (A. Ferrer), oliver@cimne.upc.edu (J.
} 


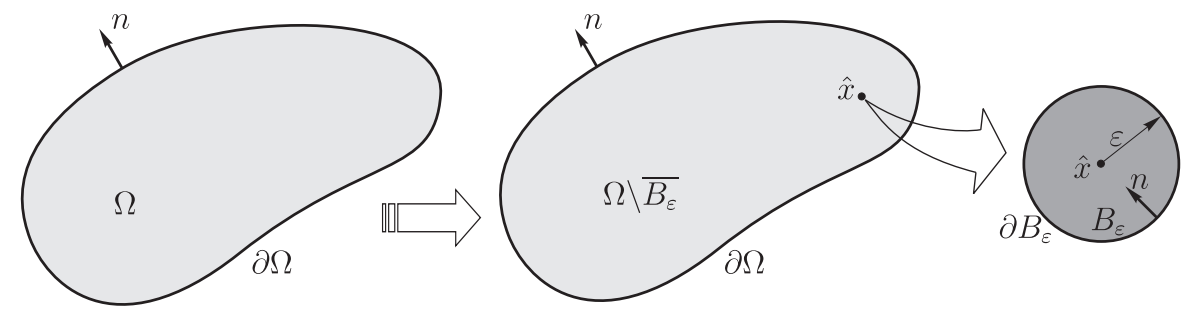

Figure 1: Topological derivative concept.

From (1), we obtain the standard definition of the topological derivative by passing to the limit $\varepsilon \rightarrow 0^{+}$:

$$
\mathcal{T}(\widehat{x})=\lim _{\varepsilon \rightarrow 0^{+}} \frac{\mathcal{J}_{\varepsilon}(\Omega)-\mathcal{J}(\Omega)}{f(\varepsilon)} .
$$

Notice that, since we are dealing with singular domain perturbations, the shape functionals $\mathcal{J}_{\varepsilon}(\Omega)$ and $\mathcal{J}(\Omega)$ are associated to topologically different domains. Therefore, the above limit is not trivial to be calculated. In particular, we need to perform an asymptotic analysis of the shape functional $\mathcal{J}_{\varepsilon}(\Omega)$ with respect to the small parameter $\varepsilon$, i.e. we need information of $\mathcal{J}_{\varepsilon}(\Omega)$ when $\varepsilon \rightarrow 0^{+}$. As it will be shown later, the shape functional difference (2) depends on the polarization tensor, which is considered a fundamental concept on the topological derivative topic. This tensor depends only on the constitutive properties of the problem and the shape of the singular domain perturbation [16, 17].

The topological derivative, in its closed form, has been fully developed for a wide range of physical phenomena. Most of them, by considering homogeneous and isotropic constitutive behaviours. In fact, only a few works dealing
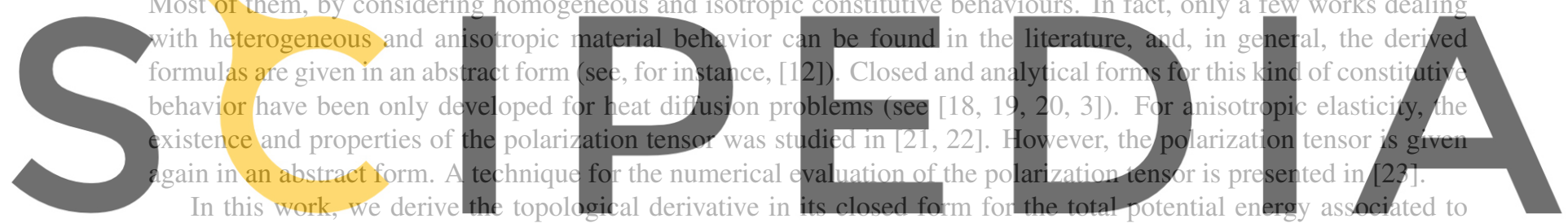

an anisotropic and heterogeneous elasticity problem. We assume as singular perturbation a small circular inclusion

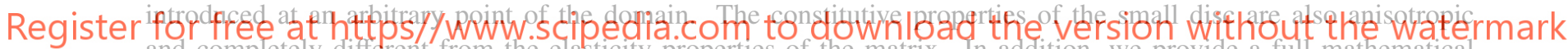
and completely different from the elasticity properties of the matrix. In addition, we provide a full mathematical justification of the derived formula, and develop precise estimates for the remainders of the topological asymptotic expansion.

Bearing this in mind, the heterogeneous anisotropic topological derivative concept, can be applied in advanced technological research areas such as topology and structural optimization simultaneously combined with topological material-design. In fact, in multi-scale modeling, for a given microstructure the homogenized constitutive response is, in general, anisotropic. In addition, since in each macroscopical structural point we have a different microstructure, the constitutive homogenized response at the macro-scale varies from point to point, i.e., it is heterogeneous. Therefore, for a correct evaluation of the topological sensitivity in a structural optimization problem, a derivative (the topological derivative) for an anisotropic and heterogeneous constitutive behaviour is needed .

This paper is organized as follows. Section 2 describes the model associated to an anisotropic and heterogeneous elasticity problem. In Section 3, we present the main result of the paper: a closed formula for the topological derivative. In Section 4, the influence of heterogeneity and anisotropy is shown through some numerical examples for structural topology optimization. The paper is closed in Section 5 where some concluding remarks are presented.

\section{Problem Formulation}

The topological asymptotic analysis of the total potential energy associated to an anisotropic and heterogeneous elasticity problem is calculated. Thus, the unperturbed shape functional is defined as:

$$
\mathcal{J}(\Omega)=\frac{1}{2} \int_{\Omega} \sigma(u) \cdot \nabla^{s} u+\int_{\Gamma_{N}} \bar{t} \cdot u
$$


where the Cauchy stress tensor $\sigma(u)$ is defined as usual:

$$
\sigma(\xi):=\mathbb{C} \nabla^{s} \xi
$$

In the above equations, $\mathbb{C}=\mathbb{C}(x)$ is a symmetric fourth order elasticity tensor, $\nabla^{s}$ is used to denote the symmetric part of the gradient operator $\nabla$ and $u$ is the displacement field, solution of the following variational problem: find the field $u \in \mathcal{U}$, such that

$$
\int_{\Omega} \sigma(u) \cdot \nabla^{s} \eta+\int_{\Gamma_{N}} \bar{t} \cdot \eta=0 \quad \forall \eta \in \mathcal{V}
$$

In the variational problem (5) the set $\mathcal{U}$ of admissible functions and the space $\mathcal{V}$ of admissible variations are given by

$$
\mathcal{U}:=\left\{\phi \in H^{1}\left(\Omega ; \mathbb{R}^{2}\right):\left.\phi\right|_{\Gamma_{D}}=\bar{u}\right\} \quad \text { and } \quad \mathcal{V}:=\left\{\phi \in H^{1}\left(\Omega ; \mathbb{R}^{2}\right):\left.\phi\right|_{\Gamma_{D}}=0\right\} .
$$

In addition, $\partial \Omega=\overline{\Gamma_{N} \cup \Gamma_{D}}$ with $\Gamma_{N} \cap \Gamma_{D}=\varnothing$, where $\Gamma_{N}$ and $\Gamma_{D}$ are Neumann and Dirichlet boundaries, respectively. Thus, $\bar{u}$ is a Dirichlet data on $\Gamma_{D}$ and $\bar{t}$ is a Neumann data on $\Gamma_{N}$, both assumed to be smooth enough, see figure 2 .

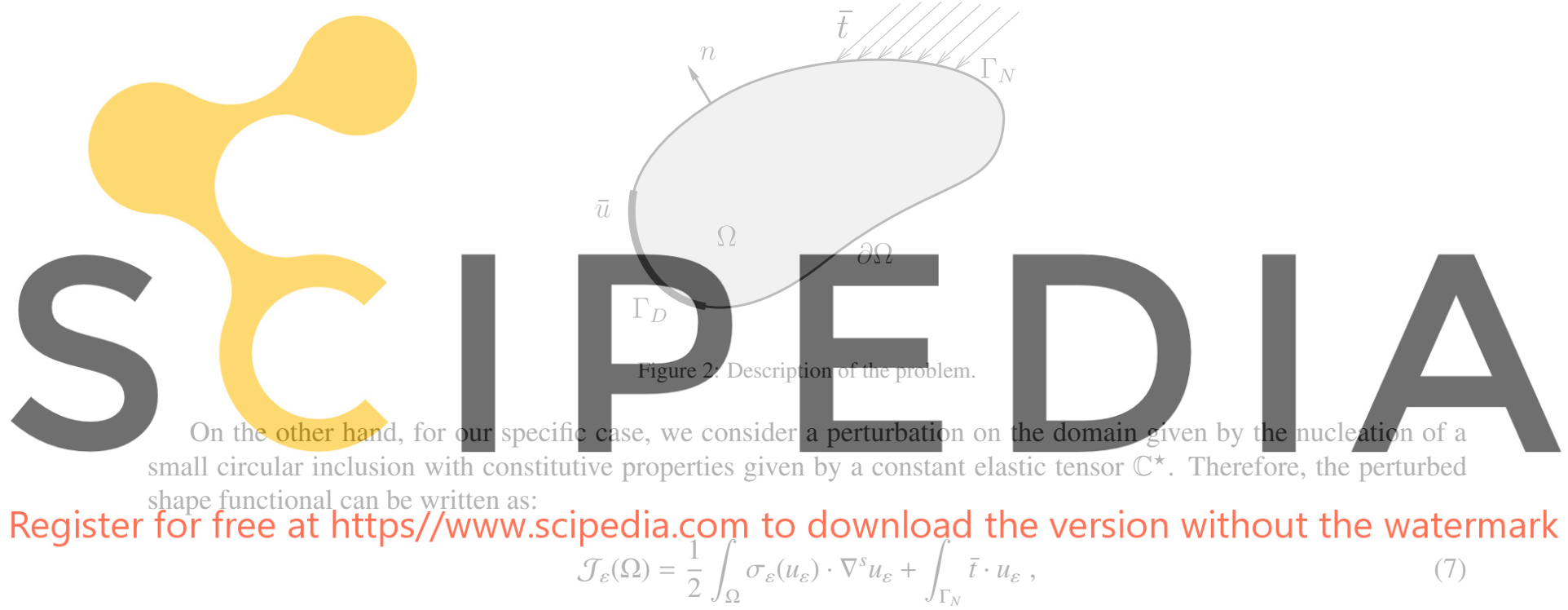

where the stress tensor associated to he perturbed configuration is defined as:

$$
\sigma_{\varepsilon}(\xi):=\mathbb{C}_{\varepsilon} \nabla^{s} \xi
$$

Here, we consider that the inclusion is made of a completely different material. Then, the elasticity tensor $\mathbb{C}_{\varepsilon}$ can be written as follows

$$
\mathbb{C}_{\varepsilon}:=\left\{\begin{array}{lll}
\mathbb{C} & \text { in } & \Omega \backslash \overline{B_{\varepsilon}} \\
\mathbb{C}^{\star} & \text { in } & B_{\varepsilon}
\end{array} .\right.
$$

In addition, in (7) the function $u_{\varepsilon}$ is solution of the following variational problem:

Find the field $u_{\varepsilon} \in \mathcal{U}_{\varepsilon}$, such that

$$
\int_{\Omega} \sigma\left(u_{\varepsilon}\right) \cdot \nabla^{s} \eta+\int_{\Gamma_{N}} \bar{t} \cdot \eta=0 \quad \forall \eta \in \mathcal{V}_{\varepsilon},
$$

and both the set $\mathcal{U}_{\varepsilon}$ and the space $\mathcal{V}_{\varepsilon}$ are defined as

$$
\mathcal{U}_{\varepsilon}:=\left\{\phi \in \mathcal{U}: \llbracket \phi \rrbracket=0 \text { on } \partial B_{\varepsilon}\right\} \quad \text { and } \quad \mathcal{V}_{\varepsilon}:=\left\{\phi \in \mathcal{V}: \llbracket \phi \rrbracket=0 \text { on } \partial B_{\varepsilon}\right\},
$$

where we use $\llbracket(\cdot) \rrbracket$ to denotes the jump of function $(\cdot)$ across the boundary $\partial B_{\varepsilon}$. Note that the domain $\Omega$ is topologically perturbed by the introduction of an inclusion $B_{\varepsilon}(\widehat{x})$ with complete different elastic constitutive properties (in general anisotropic). 


\section{Topological derivative}

In this Section we present the main result of the work: an analytical formula for the topological derivative of the energy shape functional associated to an anisotropic and heterogeneous elastic problem. We begin by choosing as admissible test function in problems (5) and (10), the function $\eta=u_{\varepsilon}-u$. Then, we obtain as consequence the following expressions

$$
\begin{aligned}
\int_{\Omega} \sigma(u) \cdot \nabla^{s} u & =\int_{\Omega} \sigma\left(u_{\varepsilon}\right) \cdot \nabla^{s} u+\int_{\Gamma_{N}} \bar{t}\left(u_{\varepsilon}-u\right), \\
\int_{\Omega} \sigma_{\varepsilon}\left(u_{\varepsilon}\right) \cdot \nabla^{s} u_{\varepsilon} & =\int_{\Omega} \sigma_{\varepsilon}\left(u_{\varepsilon}\right) \cdot \nabla^{s} u-\int_{\Gamma_{N}} \bar{t}\left(u_{\varepsilon}-u\right) .
\end{aligned}
$$

Therefore, the shape functionals (3) and (7) can be written as

$$
\begin{aligned}
\mathcal{J}(\Omega) & =\frac{1}{2} \int_{\Omega} \sigma\left(u_{\varepsilon}\right) \cdot \nabla^{s} u+\frac{1}{2} \int_{\Gamma_{N}} \bar{t}\left(u_{\varepsilon}+u\right), \\
\mathcal{J}_{\varepsilon}(\Omega) & =\frac{1}{2} \int_{\Omega} \sigma_{\varepsilon}\left(u_{\varepsilon}\right) \cdot \nabla^{s} u+\frac{1}{2} \int_{\Gamma_{N}} \bar{\tau}\left(u_{\varepsilon}+u\right) .
\end{aligned}
$$

By considering the above results, the differences of the shape functionals $\mathcal{J}_{\varepsilon}(\Omega)$ and $\mathcal{J}(\Omega)$ reads

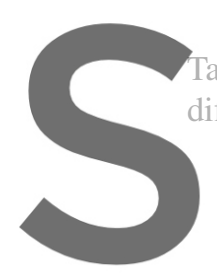

$$
\mathcal{J}_{\varepsilon}(\Omega)-\mathcal{J}(\Omega)=\frac{1}{2} \int_{\Omega} \sigma_{\varepsilon}\left(u_{\varepsilon}\right) \cdot \nabla^{s} u-\frac{1}{2} \int_{\Omega} \sigma\left(u_{\varepsilon}\right) \cdot \nabla^{s} u
$$

laking into account the definitis difference of the total potentiat

with $\Delta \mathbb{C}:=\mathbb{C}^{\star}-\mathbb{C}$.
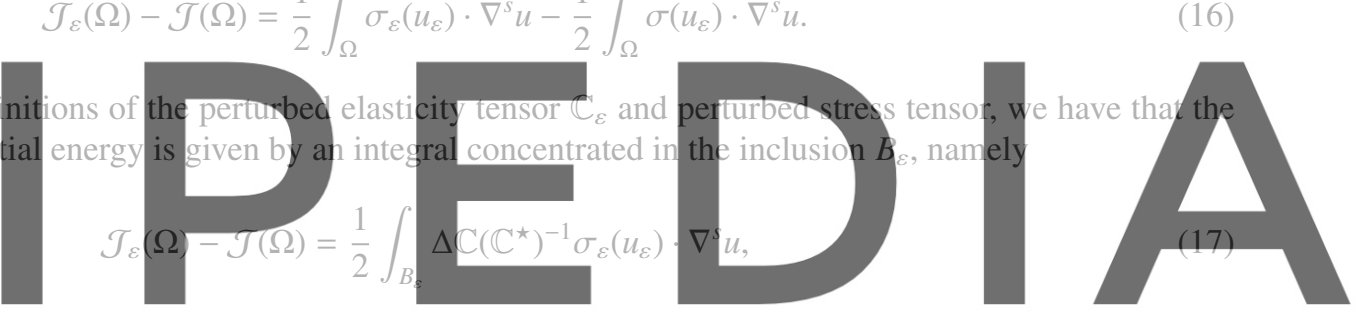

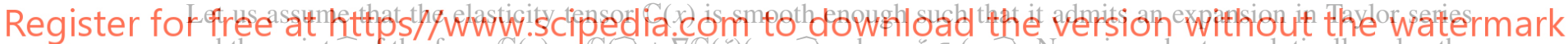
around the point $x$ of the form $\mathbb{C}(x)=\mathbb{C}(x)+\nabla \mathbb{C}(\zeta)(x-x)$, where $\zeta \in(x, x)$. Now, in order to analytically solve the integral (17), we introduce the following ansatz proposed in [24] for the solution associated to the perturbed problem $u_{\varepsilon}:$

$$
u_{\varepsilon}(x)=u(x)+\varepsilon w(x / \varepsilon)+\widetilde{u}_{\varepsilon}(x),
$$

where the function $w(y)$ is the solution of the following exterior problem independent of the small parameter $\varepsilon$

$$
\left\{\begin{array}{rlrl}
\operatorname{div}\left(\sigma_{\varepsilon}(w)\right) & =0 & \text { in } & \mathbb{R}^{2} \\
\sigma_{\varepsilon}(w) & =\mathbb{C}_{\varepsilon}(\widehat{x}) \nabla^{s} w & & \\
w & \rightarrow 0 & \text { at } & \infty \\
\llbracket w \rrbracket & =0 & \text { on } & \partial B_{1} \\
\llbracket \sigma_{\varepsilon}(w) \rrbracket n & =-\mathbb{S} \sigma(u)(\widehat{x}) n & \text { on } & \partial B_{1}
\end{array},\right.
$$

where $\mathbb{S}:=\mathbb{I}-\mathbb{C}^{\star} \mathbb{C}^{-1}, \mathbb{I}$ denote the fourth-order identity tensor and was used the change of variable $x=\varepsilon y$. The remainder $\widetilde{u}_{\varepsilon}$ in (18) must satisfy the following equation:

$$
\left\{\begin{array}{rlrl}
\operatorname{div}\left(\sigma_{\varepsilon}\left(\widetilde{u}_{\varepsilon}\right)\right) & =\varepsilon \operatorname{div}\left(\nabla \mathbb{C}_{\varepsilon}(\zeta)(x-\widehat{x}) \nabla^{s} w\right) & \text { in } & \Omega \\
\widetilde{u}_{\varepsilon} & =-\varepsilon w & \text { on } & \Gamma_{D} \\
\sigma\left(\widetilde{u}_{\varepsilon}\right) n & =-\varepsilon \sigma(w) n & \text { on } & \Gamma_{N} \\
\llbracket \widetilde{u}_{\varepsilon} \rrbracket & =0 & \text { on } & \partial B_{\varepsilon} \\
\llbracket \sigma_{\varepsilon}\left(\widetilde{u}_{\varepsilon}\right) \rrbracket n & =-\varepsilon \llbracket\left(\nabla \mathbb{C}_{\varepsilon}(\zeta) n\right)\left(\nabla^{s} u(\widehat{x})+\nabla^{s} w\right) \rrbracket n & \text { on } & \partial B_{\varepsilon}
\end{array},\right.
$$


which has the following estimate $\left\|\widetilde{u}_{\varepsilon}\right\|_{H^{1}\left(\Omega ; \mathbb{R}^{2}\right)} \leq C \varepsilon$, with the constant $C$ independent of $\varepsilon$ (see Appendix A). The exterior problem (19) is solved explicitly in Appendix B by using the complex variable method [25, 26]. From (B.17), the stress tensor $\sigma_{\varepsilon}(w)$ inside the inclusion $B_{\varepsilon}$ can be written as:

$$
\left.\sigma_{\varepsilon}(w)\right|_{B_{\varepsilon}(\widehat{x})}=\mathbb{T} \sigma(u)(\widehat{x}),
$$

where $\mathbb{T}:=-\mathbb{A} \mathbb{S}$ and the fourth order tensor $\mathbb{A}$ is shown in (B.18) of Appendix B.

Taking into account (18), the difference of shape functionals (17) reads

$$
\mathcal{J}_{\varepsilon}(\Omega)-\mathcal{J}(\Omega)=\frac{1}{2} \int_{B_{\varepsilon}} \Delta \mathbb{C}\left(\mathbb{C}^{\star}\right)^{-1}\left(\sigma_{\varepsilon}(u)+\sigma_{\varepsilon}(w)\right) \cdot \nabla^{s} u+\mathcal{E}(\varepsilon)
$$

where the term $\mathcal{E}(\varepsilon)$ is given by

$$
\mathcal{E}(\varepsilon)=\frac{1}{2} \int_{B_{\varepsilon}} \Delta \mathbb{C}\left(\mathbb{C}^{\star}\right)^{-1} \sigma_{\varepsilon}\left(\widetilde{u}_{\varepsilon}\right) \cdot \nabla^{s} u,
$$

which has the following estimate $\varepsilon(\varepsilon)=o\left(\varepsilon^{2}\right)$ as shown in the Appendix A. Next, by using the interior elliptic regularity of the function $u$ in $B_{\varepsilon}$, the difference of the shape functionals (22) satisfies the following identity:

$$
\mathcal{J}_{\varepsilon}(\Omega)-\mathcal{J}(\Omega)=\frac{1}{2} \int_{B_{\varepsilon}} \Delta \mathbb{C}(\widehat{x})\left(\mathbb{C}^{\star}\right)^{-1}\left(\sigma_{\varepsilon}(u)(\widehat{x})+\sigma_{\varepsilon}(w)\right) \cdot \nabla^{s} u(\widehat{x})+o\left(\varepsilon^{2}\right),
$$

where the expansion of the tensor $\mathbb{C}(x)$ has been used again.

With the use of (21) and the change of variables $x=\varepsilon y$ the above expression can be analytically solved leading to

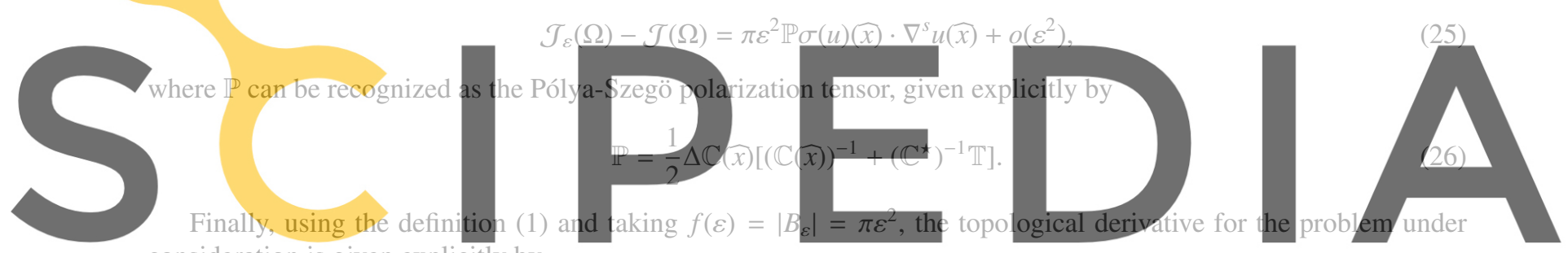

consideration is given explicitly by

\section{Register for free at https//www.scipedia.Eorm to blownlodmethe version without the watermark}

Note that this formula is general, in the sense that measures the sensitivity of the total potential energy when two materials with completely different constitutive tensors are considered. Also, the polarization tensor $\mathbb{P}$ depends only of the constitutive tensors $\mathbb{C}(\widehat{x})$ and $\mathbb{C}^{\star}$. This means that once defined $\mathbb{C}$ and $\mathbb{C}^{\star}$ for the point $\widehat{x}$, the tensor $\mathbb{P}$ can be easily obtained by computing the components of $\mathbb{A}$, see Appendix $B$, and consequently so is $\mathbb{T}$.

Remark 1. From the final expression for the polarization tensor associated to the anisotropic and heterogeneous elasticity problem (26), we can analyze the limit case when the inclusion becomes a hole by taking the limit when $\mathbb{C}^{\star} \rightarrow 0$. On the other hand, if we can analyze the sensitivity to the introduction of a rigid inclusion, we need to take the limit when $\mathbb{C}^{\star} \rightarrow \infty$. It should be noted that both limits exist and they can be easily obtained.

Remark 2. If we consider that both materials are isotropic, with constitutive properties $E$ and $v$ (background material), and $E^{\star}$ and $v^{\star}$ (inclusion), the polarization tensor (26) can be written for plane stress as a fourth-order isotropic polarization tensor, as:

$$
\mathbb{P}=-\frac{1}{2} \frac{1}{\beta \gamma+\tau_{1}}\left[(1+\beta)\left(\tau_{1}-\gamma\right) \mathbb{I}+\frac{1}{2}(\alpha-\beta) \frac{\gamma\left(\gamma-2 \tau_{3}\right)+\tau_{1} \tau_{2}}{\alpha \gamma+\tau_{2}}(I \otimes I)\right],
$$

where

$$
\alpha=\frac{1+v}{1-v}, \beta=\frac{3-v}{1+v}, \gamma=\frac{E^{\star}}{E}, \tau_{1}=\frac{1+v^{\star}}{1+v}, \tau_{2}=\frac{1-v^{\star}}{1-v} \quad \text { and } \quad \tau_{3}=\frac{v^{\star}(3 v-4)+1}{v(3 v-4)+1} .
$$

Note that by considering $v^{\star}=v$, the parameters $\tau_{i}=1$ (with $i=1 . .3$ ) and (28) becomes the polarization tensor for isotropy elasticity widely used in structural topological design [1]. 


\section{Numerical examples}

In order to evidence the potential application of the topological derivative concept for anisotropic and heterogeneous materials, some numerical examples are shown in this section.

\subsection{Numerical algorithm}

As usual in the topological optimization scientific community, a minimum compliance objective function, subject to a certain fraction volume, will be solved. To this ends, the material distribution in $\Omega$ will be identified by a characteristic function $\chi$. Thus, the objective function $\mathcal{J}(\Omega)$ can be written as a function of $\chi$ as: $\mathcal{J}\left(\Omega_{\chi}\right)$, where $\Omega_{\chi}$ is used to denote the geometrical dependency of the domain on the characteristic function $\chi$. Then, the optimization problem is:

Find the characteristic function $\chi$ such that,

$$
\begin{array}{cc}
\min _{\chi} . & \mathcal{J}\left(\Omega_{\chi}\right) \\
\text { s.t. } & c(\chi)=\int_{\Omega} \chi-V=0
\end{array}
$$

where $\mathcal{J}\left(\Omega_{\chi}\right)$ is the total potential energy of an standard elastic equilibrium problem (5) and $V$ the final intended volume. Note that the constitutive tensor $\mathbb{C}$ could be heterogeneous and anisotropic.

An efficient approach to solve such problems is the use of the level set method proposed in [27]. Some remarks must be done:

- The definition of the domain lies on the level set function $\psi$. The design variable is no longer the characteristic function $\chi$. In fact, it is parametrized by the level set $\psi$ as
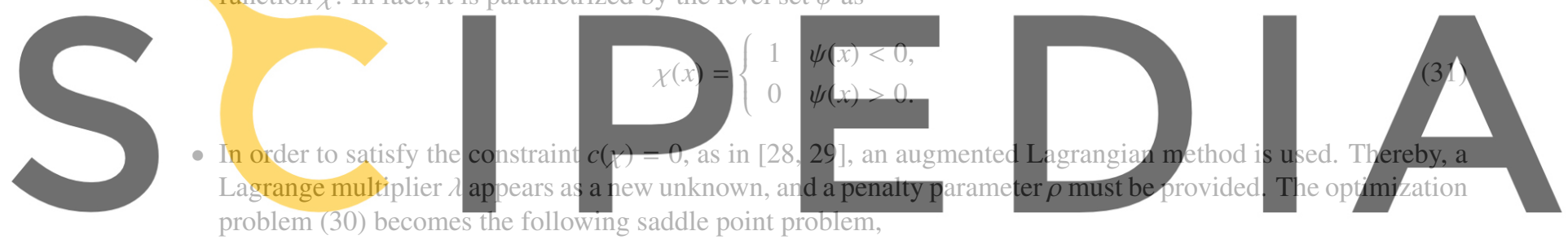

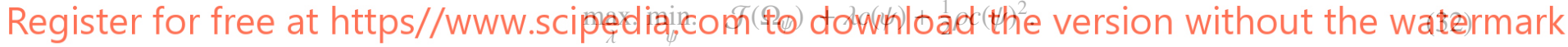

- An extended topological derivative is defined as,

$$
g(x)=\left\{\begin{array}{cc}
-\mathcal{T}(x)+\max (0, \lambda+\rho c(\psi)) & \psi(x)<0 \\
\mathcal{T}(x)+\max (0, \lambda+\rho c(\psi)) & \psi(x)>0
\end{array}\right.
$$

- The optimality condition, see [30], is based on seeking $\psi(x)$ parallel to $g(x)$ and unitary. Thus, a slerp interpolation scheme is used, i.e.,

$$
\psi_{n+1}=\frac{1}{\sin \theta_{n}}\left[\sin \left(\left(1-\kappa_{n}\right) \theta_{n}\right) \psi_{n}+\sin \left(\kappa_{n} \theta_{n}\right) \frac{g_{n}}{\left\|g_{n}\right\|_{L^{2}}}\right],
$$

- $\kappa_{n} \in[0,1]$ is a line search-like parameter and the converge criterion is based on the value of $\theta_{n}$, the angle between $\psi_{n}$ and $g_{n}$, which is written as

$$
\theta_{n}=\operatorname{acos}\left[\frac{\left(\psi_{n}, g_{n}\right)}{\|\psi\|_{L^{2}}\left\|g_{n}\right\|_{L^{2}}}\right] .
$$

Note that, in this fix point scheme, the topological derivative for anisotropic materials plays the role of the gradient in an steepest descent algorithm. With this algorithm, some numerical examples, both with homogeneous and heterogenous material distribution, are considered.All them are solved under 2D elastic plane stress assumptions. Regarding to numerical aspects, a standard FEM is used for solving (5). 


\subsection{Representative Numerical Simulations}

In this first set of case studies, the constitutive behaviour is homogeneous in the design domain. Besides, the constitutive tensor in the inclusion is defined with the contrast parameter $\gamma$ as follows: $\mathbb{C}^{\star}=\gamma \mathbb{C}$ with $\gamma=10^{-4}$. The $2 \times 1$ domain is discretized with a structured mesh of $5200 \mathrm{P} 1$ triangular elements. The volume fraction is taken as $V=0.4$ and the penalty as $\rho=0.5$. All the examples are declared to be converged when $\theta<1^{\circ}$ and $|c(\psi)|<0.001$.

\subsubsection{Homogeneous material distribution - Traction rod}

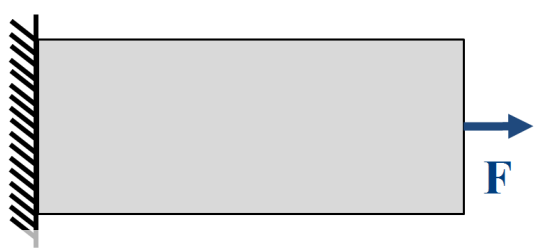

As shown in Figure 3, regarding to boundary conditions, the domain is fixed at the left side and has a horizontal unitary force at the middle of the right send. Some representative cases, in terms of the definition of the constitutive tensor, have been selected (see second column in Figure 4).
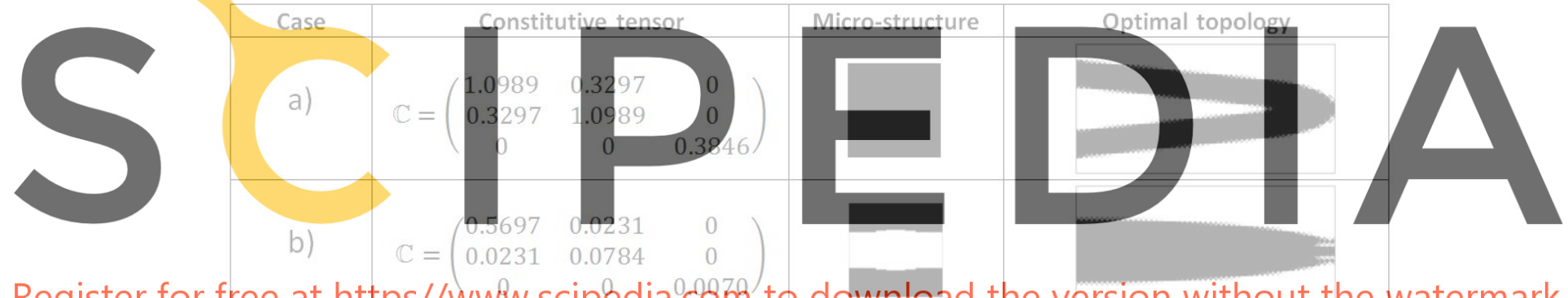

Register for free at https//www.scipedia.coth to download the version without the watermark

\begin{tabular}{|l|l} 
c) & $\mathbb{C}=\left(\begin{array}{ccc}0.0784 & 0.0231 & 0 \\
0.0231 & 0.5697 & 0 \\
0 & 0 & 0.0070\end{array}\right)$ \\
\hline d) & $\mathbb{C}=\left(\begin{array}{ccc}0.2867 & 0.1900 & 0 \\
0.1900 & 0.2867 & 0 \\
0 & 0 & 0.1715\end{array}\right)$ \\
\hline e) & $\mathbb{C}=\left(\begin{array}{ccc}0.3400 & 0.1689 & 0 \\
0.1689 & 0.3400 & 0 \\
0 & 0 & 0.1401\end{array}\right)$ \\
\hline f) & $\mathbb{C}=\left(\begin{array}{ccc}0.2479 & 0.1208 & 0.1178 \\
0.1208 & 0.2050 & 0.0613 \\
0.1178 & 0.0613 & 0.1391\end{array}\right)$ \\
\hline
\end{tabular}

Figure 4: Representative optimal homogeneous traction rod topologies: (a) Isotropic ( $E=1$ and $v=0.3$ ) as a reference, (b)-(e) Orthotropic, (f) Anisotropic 
In the second column, the constitutive tensor used is shown, which is obtained by a classical homogenization procedure of the micro-structure displayed on the third column, see [31]. In the fourth one, the final optimal topology for the structure is also shown.

Notice the strong influence of the selected micro-structure topology and, consequently, of the resulting homogenized constitutive tensor, on the obtained optimal macro-structure topology.

\subsubsection{Homogeneous material distribution - Cantilever beam}
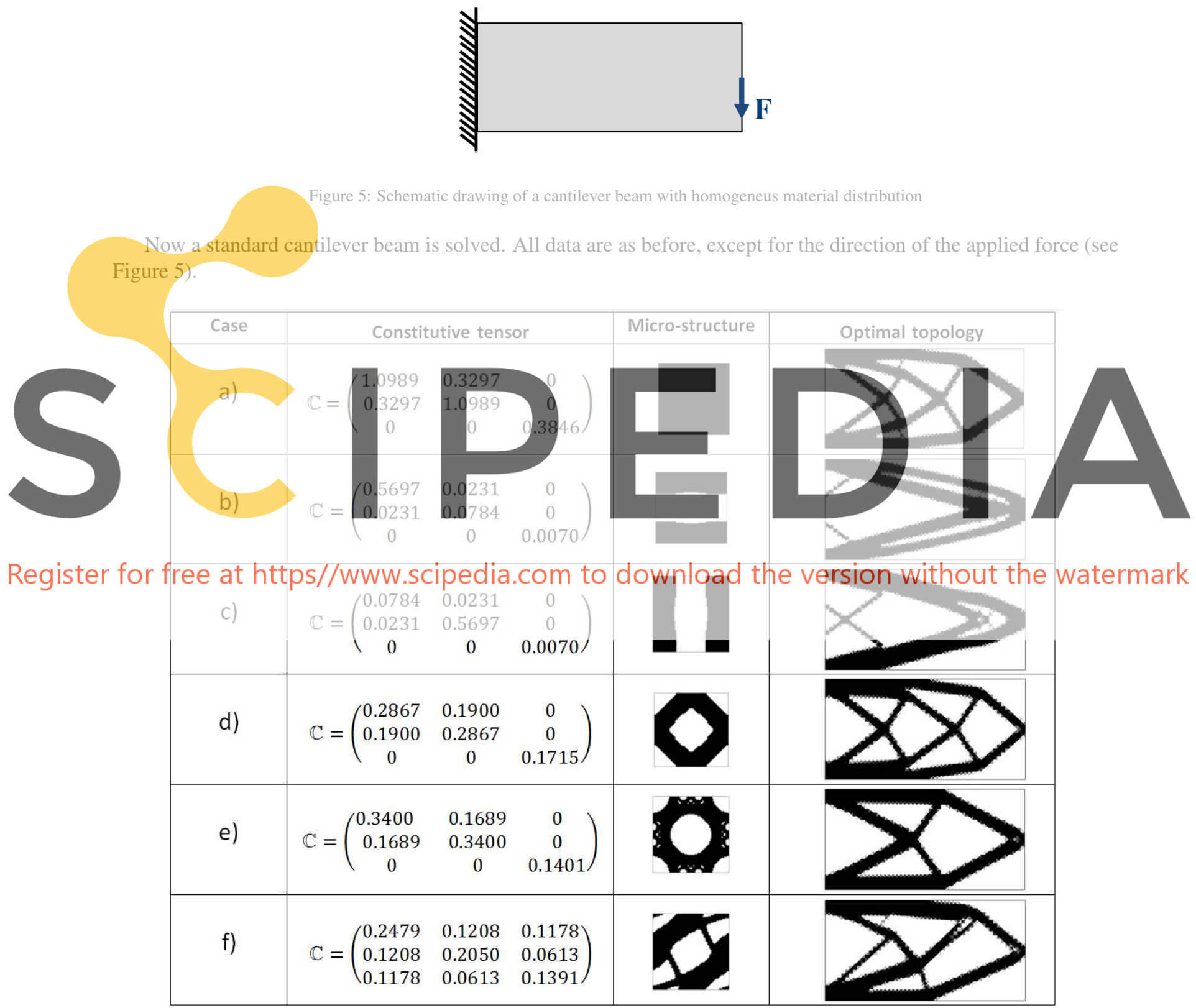

Figure 6: Representative optimal homogeneous traction rod topologies: (a) Isotropic ( $E=1$ and $v=0.3$ ) as a reference, (b)-(e) Orthotropic, (f) Anisotropic

The isotropic case is used also as a reference. Some unconventional topologies are obtained, specially in the 
orthotropic case (micro-structure with horizontal and vertical bars) and full anisotropic case (last row). It can be observed, that the resulting macro-structure topology, tends to arrange following the principal directions of the microstructure topology.

\subsubsection{Heterogeneous material distribution}

In the following set of examples, an heterogeneous distribution of material is used for a classical cantilever beam optimal design. The contrast parameter is taken $\gamma=10^{-4}$. The $2 \times 1$ domain is discretized through a structured mesh of $6272 \mathrm{P} 1$ elements. The geometry is vertically partitioned in four domains with the same width (1/4). The top and bottom regions are endowed with a constitutive tensor different from the center one (see Figures 7 and 9). The intended volume fraction is $V=0.4$ and the considered penalty value is $\rho=0.5$. Again the iterative solution algorithm is declared converged when $\theta<1^{\circ}$ and $|c(\psi)|<0.001$.

- Case 1. In this example, the top and bottom regions are endowed with a microstructure topology yielding horizontallydominant microscopic stiffness, where the center region, microscopic topology enforces shear-dominant stiffness.

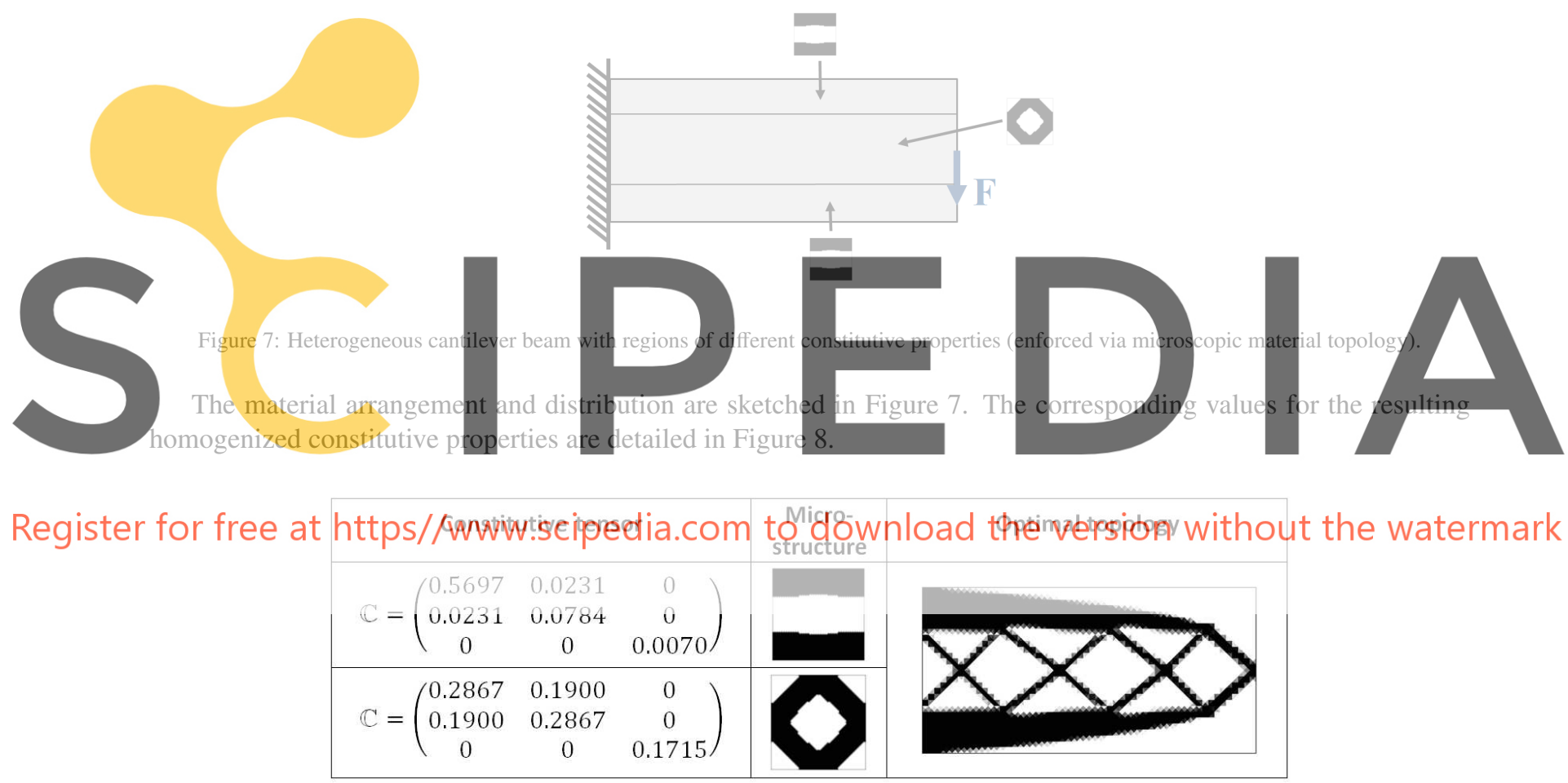

Figure 8: Heterogeneous cantilever beam. Material properties and obtained results (case 1).

It is worth noting that both constitutive tensors are again anisotropic. In addition, note that the optimal topology with the anisotropic heterogenous material distribution is quite different from the homogeneous isotropic case (first row of Figure 6) and the homogeneous anisotropic case (last row of Figure 6). Again, it can be observed as the macroscopic topology tends to mimic the microscopic one in the different considered regions.

- Case 2. Now the top and bottom regions are endowed with a microstructure yielding horizontally-dominant elastic stiffness, whereas the central region is endowed with a vertically-dominant one.

Figure 9 shows an schematic picture of this case. Details of the resulting homogenized elastic properties are given in Figure 10. 


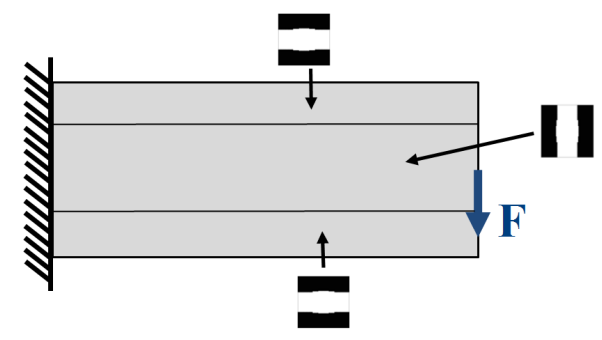

Figure 9: Heterogeneous cantilever beam. Material properties and obtained results (case 2)

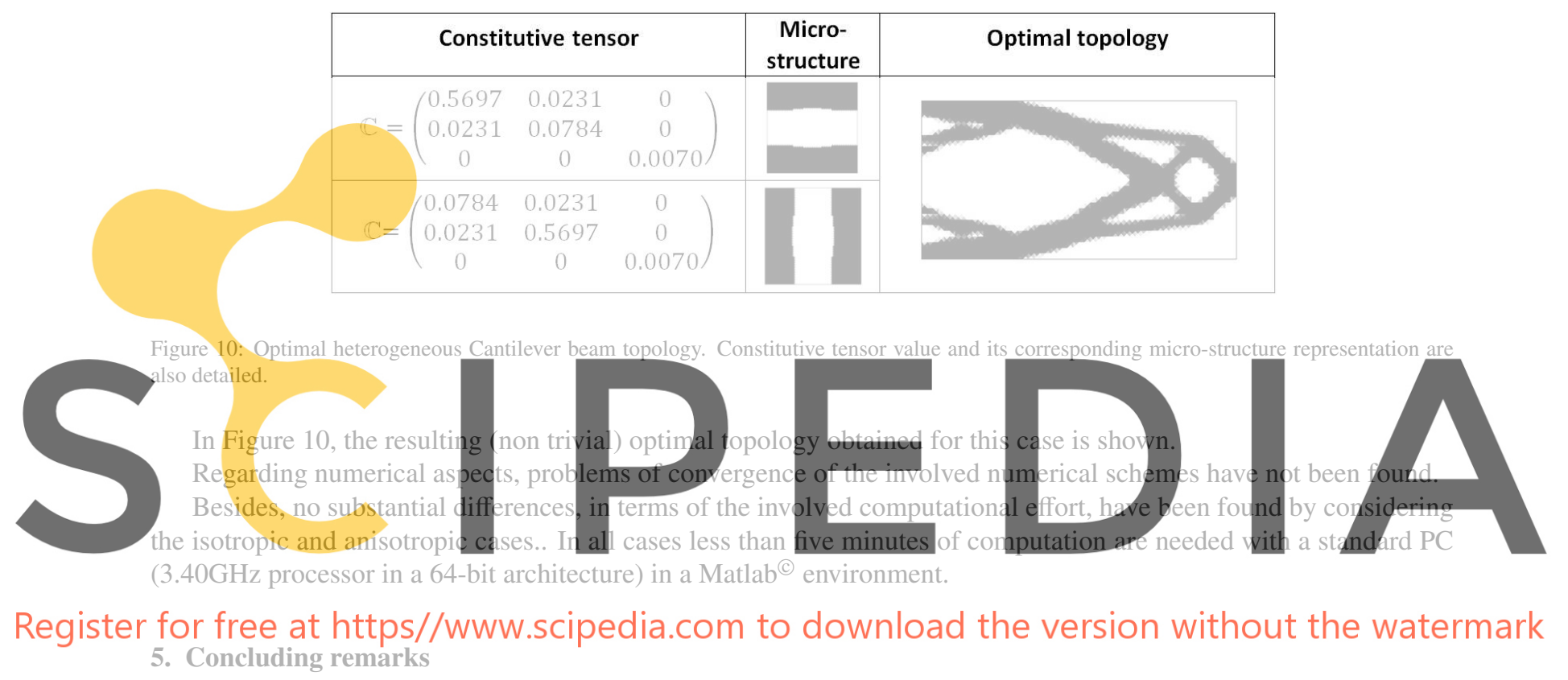

In this work an analytical and closed-form expression for the topological derivative in heterogeneous and anisotropic elastic problems has been presented. We consider as singular perturbation the introduction of a circular disc, with an anisotropic constitutive tensor completely different from the background material, in an arbitrary point. From the asymptotic analysis, it has been proven that the heterogeneous behavior of the material properties does not contribute to the first order topological derivative. The polarization tensor for this problem is obtained from a very simple (and inexpensive) matrix evaluation, in terms of the constitutive properties of the unperturbed problem and the inclusion. The derived formula are general for any kind of anisotropy in two dimensional problems, where orthotropy and isotropy (of the background material or the inclusion or a combination of both) can be derived as particular cases.

We recall that the development presented in this work for the total potential energy, in particular the solution of the so-called exterior problem, can be applied for other cost functionals and that the derivation of the associated topological derivative can be easily done.

With theses results in mind, the presented numerical simulation show that the selected material properties heavily affect the optimal topology in an standard topology optimization problem. Since real applications involve nonhomogenous isotropic material, the obtained closed formula of the topological derivative for heterogeneous anisotropic materials generalizes the concept of topology optimization for any kind of material properties and distribution.

In addition, a certain influence of the microscopic topology on the optimal macroscopic topology is observed which, in some cases, becomes very relevant. This suggests a possible approach, based on the use of the anisotropic topological derivative, to link the microscopic and macroscopic topologies in optimal topological designs. This issue is left for future research. 


\section{Acknowledgments}

The research leading to these results has received funding from the European Research Council under the European Union's Seventh Framework Programme (FP/2007-2013) / ERC Grant Agreement n. 320815 (ERC Advanced Grant Project "Advanced tools for computational design of engineering materials" COMP-DES-MAT). The work of S.M. Giusti was partially supported by Argentinean Agencies: ANPCyT (National Agency for Scientific and Technical Promotion), PID-UTN (Research and Development Program of the National Technological University) and CONICET (National Council for Scientific and Technical Research), under grants N PICT 2014-3372, PID/UTN 2050-2013 and PIP 2013-631, respectively. The support of all these agencies is gratefully acknowledged.

\section{Appendix A. Estimation of the remainders}

In this Section the estimation of the remainders in the topological asymptotic expansion is performed. This estimations has been used in the derivation of the topological derivative expression (27) presented in Section 3 . In particular, we study the asymptotic behavior of the remainder $\widetilde{u}_{\varepsilon}$ in (20) and the residue $\mathcal{E}(\varepsilon)$ defined in (23). Let us start by introducing the following lemma that ensures the existence of the topological derivative for the problem under analysis:

Lemma 3. Let $u$ and $u_{\varepsilon}$ be solutions to (5) and (10), respectively. Then, we have that the estimate $\left\|u_{\varepsilon}-u\right\|_{H^{1}\left(\Omega ; \mathbb{R}^{2}\right)}=$ $O(\varepsilon)$ holds true.

Proof. We start by subtracting the variational problem (5) and (10) to obtain:

$$
\int_{\Omega} \sigma_{\varepsilon}\left(u_{\varepsilon}-u\right) \cdot \nabla^{s} \eta=\int_{B_{\varepsilon}} \mathbb{S} \sigma(u) \cdot \nabla^{s} \eta .
$$

with $\mathbb{S}=\mathbb{I}-\mathbb{C}^{\star} \mathbb{C}^{-1}$. Now, by taking $\eta=u_{\varepsilon}-u$ as test function in the above equation, we obtain the following equality:

$$
\int_{\Omega} \sigma_{\varepsilon}\left(u_{\varepsilon}-u\right) \cdot \nabla^{s}\left(u_{\varepsilon}-u\right)=\int_{B_{\varepsilon}} \mathbb{S} \sigma(u) \cdot \nabla^{s}\left(u_{\varepsilon}-u\right) .
$$

From the Cauchy-Schwartz and Poincaré inequality it follows that

$$
\begin{aligned}
\int_{\Omega} \sigma_{\varepsilon}\left(u_{\varepsilon}-u\right) \cdot \nabla^{s}\left(u_{\varepsilon}-u\right) & \leq C_{1}\|\sigma(u)\|_{L^{2}\left(B_{\varepsilon} ; \mathbb{R}^{2}\right)}\left\|\nabla^{s}\left(u_{\varepsilon}-u\right)\right\|_{L^{2}\left(B_{\varepsilon} ; \mathbb{R}^{2}\right)} \\
& \leq C_{2} \varepsilon\left\|\nabla^{s}\left(u_{\varepsilon}-u\right)\right\|_{L^{2}\left(B_{\varepsilon} ; \mathbb{R}^{2}\right)} \\
& \leq C_{3} \varepsilon\left\|\nabla^{s}\left(u_{\varepsilon}-u\right)\right\|_{H^{1}\left(\Omega ; \mathbb{R}^{2}\right)} \\
& \leq C_{4} \varepsilon\left\|u_{\varepsilon}-u\right\|_{H^{1}\left(\Omega ; \mathbb{R}^{2}\right)},
\end{aligned}
$$

where we have used the elliptic regularity of function $u$. Finally, from the coercivity of the bilinear form of (10), namely

$$
c\left\|u_{\varepsilon}-u\right\|_{H^{1}\left(\Omega ; \mathbb{R}^{2}\right)}^{2} \leq \int_{\Omega} \sigma_{\varepsilon}\left(u_{\varepsilon}-u\right) \cdot \nabla^{s}\left(u_{\varepsilon}-u\right),
$$

we obtain the result with the constant $C_{4} / c$ independent of the small parameter $\varepsilon$.

Lemma 4. Let $\widetilde{u}_{\varepsilon}$ be solution to (20). Then, the following estimate holds true:

$$
\left\|\widetilde{u}_{\varepsilon}\right\|_{H^{1}\left(\Omega ; \mathbb{R}^{2}\right)} \leq C \varepsilon,
$$

with the constant $C$ independent of the small parameter $\varepsilon$. 
Proof. From the ansatz proposed in (18) for $u_{\varepsilon}$ and making use of the triangular inequality, we obtain:

$$
\begin{aligned}
\left|\widetilde{u}_{\varepsilon}\right|_{H^{1}\left(\Omega ; \mathbb{R}^{2}\right)} & =\left|u_{\varepsilon}-u-\varepsilon w\right|_{H^{1}\left(\Omega ; \mathbb{R}^{2}\right)} \\
& \leq\left|u_{\varepsilon}-u\right|_{H^{1}\left(\Omega ; \mathbb{R}^{2}\right)}+\varepsilon|w|_{H^{1}\left(\Omega ; \mathbb{R}^{2}\right)} \\
& \leq\left\|u_{\varepsilon}-u\right\|_{H^{1}\left(\Omega ; \mathbb{R}^{2}\right)}+\varepsilon|w|_{H^{1}\left(\mathbb{R}^{2} ; \mathbb{R}^{2}\right)} \\
& \leq C_{1} \varepsilon
\end{aligned}
$$

where we have used the change of variables $x=\varepsilon y$, the equivalence between the semi-norm and the norm in $H^{1}\left(\Omega ; \mathbb{R}^{2}\right)$ and the estimate in Lemma 3. Finally, the results comes out from the Poincaré inequality.

Lemma 5. Let $\widetilde{u}_{\varepsilon}$ and $u$ be solutions to (20) and (5), respectively. Then, we have the following estimate for the remainder $\mathcal{E}(\varepsilon)$ in $(23)$ :

$$
\frac{1}{2} \int_{B_{\varepsilon}} \Delta \mathbb{C}\left(\mathbb{C}^{\star}\right)^{-1} \sigma_{\varepsilon}\left(\widetilde{u}_{\varepsilon}\right) \cdot \nabla^{s} u=o\left(\varepsilon^{2}\right)
$$

Proof. From the Cauchy-Schwartz inequality we obtain

$$
\begin{aligned}
\mathcal{E}(\varepsilon) & =\frac{1}{2} \int_{B_{\varepsilon}} \Delta \mathbb{C}\left(\mathbb{C}^{\star}\right)^{-1} \sigma_{\varepsilon}\left(\widetilde{u}_{\varepsilon}\right) \cdot \nabla^{s} u \\
& \leq C_{1}\left\|\nabla^{s} u\right\|_{L^{2}\left(B_{\varepsilon} ; \mathbb{R}^{2}\right)}\left\|\nabla^{s} \widetilde{u}_{\varepsilon}\right\|_{L^{2}\left(B_{\varepsilon} ; \mathbb{R}^{2}\right)} \\
& \leq \varepsilon C_{2}\left\|\nabla^{s} \widetilde{u}_{\varepsilon}\right\|_{L^{2}\left(B_{\varepsilon} ; \mathbb{R}^{2}\right)}
\end{aligned}
$$

Note that from problem (20) that the r.h.s. depends explicitly on the small parameter $\varepsilon$. Therefore, since this problem is linear and in view of Lemma 4 , we can write $\widetilde{u}_{\varepsilon}=\varepsilon v_{0}$. Then, we have

$$
\begin{aligned}
\mathcal{E}(\varepsilon) & \leq \varepsilon^{2} C_{3}\left\|\nabla^{s} v_{0}\right\|_{L^{2}\left(B_{\varepsilon} ; \mathbb{R}^{2}\right)} \\
& \leq \varepsilon^{3} C_{4} .
\end{aligned}
$$

which leads to the result.

\section{Appendix B. Solution of the exterior problem}

In this Section we show the analytical solution of the problem presented in (19). In particular, we present the solution of a generalized version of problem (19), namely,

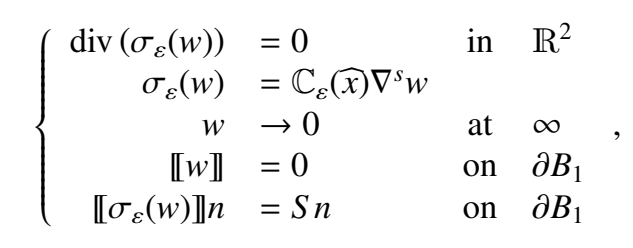

where $S$ is a symmetric second order tensor.

A general methodology to solve problem (B.1) can be constructed by using the complex variable method for anisotropic elasticity [25, 26]. Now we briefly present the main steps to solve the above problem. A complete description of the methodology and its application can be found in the books [25, 26] and [32].

First we define a new coordinate system $z_{i}=x+\mu_{i} y$ for $i=1 . .2$, where $\mu_{i}$ are complex parameters, roots of the following equation:

$$
\alpha_{11} \mu^{4}-2 \alpha_{13} \mu^{3}+\left(2 \alpha_{12}+\alpha_{33}\right) \mu^{2}-2 \alpha_{23} \mu+\alpha_{22}=0
$$

where $\alpha_{i j}$, with $(i, j)=1 . .3$, are the components of $\mathbb{C}^{-1}$ (in matrix notation). Two cases are possible, depending on the relations between the elastic constant: (i) the roots are all different: $\mu_{1}, \mu_{2}, \bar{\mu}_{1}$ and $\bar{\mu}_{2}$; and (ii) the roots are pairwise 
equal: $\mu_{1}=\mu_{2}$ and $\bar{\mu}_{1}=\bar{\mu}_{2}$. Here, $\bar{\mu}_{1}$ and $\bar{\mu}_{2}$ denotes the complex conjugated of parameters $\mu_{1}$ and $\mu_{2}$. Based on the previous definitions, a general expression for the stress components can be obtained as:

$$
\begin{aligned}
& \left(\sigma_{\varepsilon}(w)\right)_{x x}=2 \mathfrak{R}\left[\mu_{1}^{2} \Phi_{1}^{\prime}\left(z_{1}\right)+\mu_{2}^{2} \Phi_{2}^{\prime}\left(z_{2}\right)\right], \\
& \left(\sigma_{\varepsilon}(w)\right)_{y y}=2 \mathfrak{R}\left[\Phi_{1}^{\prime}\left(z_{1}\right)+\Phi_{2}^{\prime}\left(z_{2}\right)\right], \\
& \left(\sigma_{\varepsilon}(w)\right)_{x y}=-2 \mathfrak{R}\left[\mu_{1} \Phi_{1}^{\prime}\left(z_{1}\right)+\mu_{2} \Phi_{2}^{\prime}\left(z_{2}\right)\right],
\end{aligned}
$$

with $\mathfrak{R}[\bullet]$ denoting the real part of $[\bullet]$ and the prime is used to denote the derivative for the function $f(z)$ respect to the variable $z$, i.e. $f^{\prime}(z)=\partial f / \partial z$. Also, the displacement components are written in term of the complex potentials $\Phi_{1}$ and $\Phi_{2}$ as:

$$
\begin{aligned}
& (w)_{x}=2 \mathfrak{R}\left[p_{1} \Phi_{1}\left(z_{1}\right)+p_{2} \Phi_{2}\left(z_{2}\right)\right]-\omega y, \\
& (w)_{y}=2 \mathfrak{R}\left[q_{1} \Phi_{1}\left(z_{1}\right)+q_{2} \Phi_{2}\left(z_{2}\right)\right]+\omega x,
\end{aligned}
$$

where $\omega$ characterize a rotation and the complex numbers $p_{i}$ and $q_{i}$ are:

$$
\begin{aligned}
p_{i} & =\alpha_{11} \mu_{i}^{2}+\alpha_{12}-\alpha_{13} \mu_{i}, \\
q_{i} & =\alpha_{12} \mu_{i}+\alpha_{22} / \mu_{i}-\alpha_{23}, \quad i=1,2 .
\end{aligned}
$$

The complex potentials $\Phi_{1}$ and $\Phi_{2}$ are related to the stress function $F(x, y)$ by means of the relations:

$$
\Phi_{1}=\frac{\partial F}{\partial z_{1}} \quad \text { and } \quad \Phi_{2}=\frac{\partial F}{\partial z_{2}} .
$$

In order to facilitate the notation, in the sequel we use the following definitions:

$$
\sigma_{\varepsilon}^{e}:=\left.\sigma_{\varepsilon}(w)\right|_{\Omega \backslash \overline{B_{\varepsilon}}} \text { and } \sigma_{\varepsilon}^{i}:=\left.\sigma_{\varepsilon}(w)\right|_{B_{\varepsilon}} .
$$

Now, by applying the traction boundary condition on $\partial B_{\varepsilon}$, see (B.1 $)_{5}$, and in view of the fact that the stress inside of the inclusion $B_{\varepsilon}$ is constant, see $[33,34,35]$, the complex potential for the solution outside $B_{\varepsilon}$, can be written as:

$$
\begin{aligned}
& \Phi_{1}\left(z_{1}\right)=\frac{1}{2\left(\mu_{1}-\mu_{2}\right)}\left[\left(\left(\sigma_{\varepsilon}^{i}\right)_{x x}+S_{x x}\right) i-\left(\left(\sigma_{\varepsilon}^{i}\right)_{x y}+S_{x y}\right)\left(1-i \mu_{2}\right)-\left(\left(\sigma_{\varepsilon}^{i}\right)_{y y}+S_{y y}\right) \mu_{2}\right] \frac{1}{\zeta_{1}} \\
& \Phi_{2}\left(z_{2}\right)=-\frac{1}{2\left(\mu_{1}-\mu_{2}\right)}\left[\left(\left(\sigma_{\varepsilon}^{i}\right)_{x x}+S_{x x}\right) i-\left(\left(\sigma_{\varepsilon}^{i}\right)_{x y}+S_{x y}\right)\left(1-i \mu_{1}\right)-\left(\left(\sigma_{\varepsilon}^{i}\right)_{y y}+S_{y y}\right) \mu_{1}\right] \frac{1}{\zeta_{2}} .
\end{aligned}
$$

where $S_{i j}$ and $\left(\sigma_{\varepsilon}^{i}\right)_{i j}$ are the components of tensors $S$ and $\sigma_{\varepsilon}^{i}$, respectively, measured in the coordinate system $(x, y)$, and

$$
\zeta_{k}=\frac{z_{k}+\sqrt{z_{k}^{2}-1-\mu_{k}^{2}}}{1-i \mu_{k}} \quad \text { with } \quad k=1,2 .
$$

Note that expression (B.8) indicates that the stress field outside the inclusion $B_{\varepsilon}$ can be explicitly obtained in terms of the boundary condition on $\partial B_{\varepsilon}$ and the stress inside $B_{\varepsilon}$. The components of $\sigma_{\varepsilon}^{e}$ can be obtained by inserting the potentials (B.8) in (B.3).

Next, by applying the displacement boundary condition (B.1) $)_{4}$, see ([26], Chapter VI, Section 41), we obtain the following system of equations, which will define the four unknown: components of the stress $\sigma_{\varepsilon}^{i}$ and the rotation of the inclusion $\omega$,

$$
K s=F s_{m},
$$


where matrices $K$ and $F$; and vectors $s$ and $s_{m}$ are given by:

$$
\begin{aligned}
& K=\left(\begin{array}{cccr}
-\left(\mathfrak{I}[\kappa]+\alpha_{11}^{\star}\right) & -\left(\mathfrak{R}[\lambda]+\alpha_{12}^{\star}\right) & -\left(\mathfrak{R}[\kappa]+\mathfrak{J}[\lambda]+\alpha_{13}^{\star}\right) & 0 \\
\mathfrak{R}[\kappa] & -\mathfrak{I}[\lambda] & \mathfrak{R}[\lambda]-\mathfrak{I}[\kappa] & 1 \\
-\left(\mathfrak{I}[\gamma]+\alpha_{13}^{\star}\right) & -\left(\mathfrak{R}[\rho]+\alpha_{23}^{\star}\right) & -\left(\mathfrak{R}[\gamma]+\mathfrak{I}[\rho]+\alpha_{33}^{\star}\right) & -1 \\
\mathfrak{R}[\gamma]-\alpha_{12}^{\star} & -\left(\mathfrak{J}[\rho]+\alpha_{22}^{\star}\right) & \mathfrak{R}[\rho]-\mathfrak{I}[\gamma]-\alpha_{32}^{\star} & 0
\end{array}\right) \\
& s=\left(\begin{array}{c}
\left(\sigma_{\varepsilon}^{i}\right)_{x x} \\
\left(\sigma_{\varepsilon}^{i}\right)_{y y} \\
\left(\sigma_{\varepsilon}^{i}\right)_{x y} \\
\omega
\end{array}\right) \\
& F=\left(\begin{array}{rrr}
\mathfrak{I}[\kappa] & \mathfrak{R}[\lambda] & \mathfrak{J}[\lambda]+\mathfrak{R}[\kappa] \\
-\mathfrak{R}[\kappa] & \mathfrak{J}[\lambda] & -\mathfrak{R}[\lambda]+\mathfrak{I}[\kappa] \\
\mathfrak{I}[\gamma] & \mathfrak{R}[\rho] & \mathfrak{I}[\rho]+\mathfrak{R}[\gamma] \\
-\mathfrak{R}[\gamma] & \mathfrak{J}[\rho] & -\mathfrak{R}[\rho]+\mathfrak{I}[\gamma]
\end{array}\right) \\
& s_{m}=\left(\begin{array}{c}
S_{x x} \\
S_{y y} \\
S_{x y}
\end{array}\right)
\end{aligned}
$$

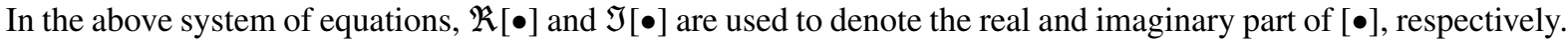
Also, $\alpha_{i j}^{\star}$, with $(i, j)=1 . .3$, are the components of $\left(\mathbb{C}^{\star}\right)^{-1}$ (in matrix notation) and the complex number $\rho, \lambda, \gamma$ and $\kappa$ are given by:

$$
\rho=\frac{q_{1} \mu_{2}-q_{2} \mu_{1}}{\mu_{1}-\mu_{2}}, \quad \gamma=\frac{q_{1}-q_{2}}{\mu_{1}-\mu_{2}}, \quad \kappa=\frac{p_{1}-p_{2}}{\mu_{1}-\mu_{2}} \quad \text { and } \quad \lambda=\frac{p_{1} \mu_{2}-p_{2} \mu_{1}}{\mu_{1}-\mu_{2}} .
$$

Then, in view of the system (B.10) the stress inside of the inclusion can be obtained as:

$$
\left(\begin{array}{c}
\left(\sigma_{\varepsilon}^{i}\right)_{x x} \\
\left(\sigma_{\varepsilon}^{i}\right)_{y y} \\
\left(\sigma_{\varepsilon}^{i}\right)_{x y}
\end{array}\right)=A s_{m}
$$

where matrix $A$ is given by the first three rows of $K^{-1} F$. The components of matrix $A$ are not explicitly given here due to their complexity.

Finally, by rearranging (B.16), the solution of (B.1) in $B_{\varepsilon}$ can be written in tensorial notation as a function of the boundary condition in $\partial B_{\varepsilon}$ :

$$
\left.\sigma_{\varepsilon}(w)\right|_{B_{\varepsilon}}=\mathbb{A} S \text {, }
$$

where components of the tensor $\mathbb{A}$ is related to components of the matrix $A$ by the standard contracted notation (or Voigt notation) using the following rules for replace the subscript:

$$
11 \rightarrow 1, \quad 22 \rightarrow 2 \text { and } 12 \rightarrow 3
$$

In view of the symmetries of $\sigma_{\varepsilon}(w)$ and $S$, the tensor $\mathbb{A}$ has the follows symmetry properties:

$$
\mathbb{A}_{i j k l}=\mathbb{A}_{j i k l} \quad \text { and } \quad \mathbb{A}_{i j k l}=\mathbb{A}_{i j l k}
$$

\section{References}

[1] A. A. Novotny, J. Sokołowski, Topological derivatives in shape optimization, Interaction of Mechanics and Mathematics, Springer-Verlag, Berlin, Heidelberg, 2013.

[2] J. Sokołowski, A. Zochowski, Topological derivative for optimal control problems, Control and Cybernetics 28 (3) (1999) 611-626.

[3] J. Sokołowski, A. Żochowski, On the topological derivative in shape optimization, SIAM Journal on Control and Optimization 37 (4) (1999) $1251-1272$.

[4] R. C. R. Amigo, S. Giusti, A. A. Novotny, E. C. N. Silva, J. Sokolowski, Optimum design of flextensional piezoelectric actuators into two spatial dimensions, SIAM Journal on Control and Optimization 52 (2) (2016) 760-789.

[5] S. Amstutz, A. A. Novotny, E. A. de Souza Neto, Topological derivative-based topology optimization of structures subject to Drucker-Prager stress constraints, Computer Methods in Applied Mechanics and Engineering 233-236 (2012) 123-136. 
[6] S. Amstutz, A. A. Novotny, N. Van Goethem, Minimal partitions and image classification using a gradient-free perimeter approximation, Inverse Problems and Imaging 8 (2) (2014) 361-387.

[7] J. R. de Faria, D. Lesnic, Topological derivative for the inverse conductivity problem: A bayesian approach, Journal of Scientific Computing 63 (1) (2015) 256-278.

[8] S. M. Giusti, A. A. Novotny, E. A. de Souza Neto, R. A. Feijóo, Sensitivity of the macroscopic elasticity tensor to topological microstructural changes, Journal of the Mechanics and Physics of Solids 57 (3) (2009) 555-570.

[9] A. Khludnev, J. Sokołowski, K. Szulc, Shape and topological sensitivity analysis in domains with cracks, Applications of Mathematics 55 (6) (2010) 433-469.

[10] L. F. N. Sá, R. C. R. Amigo, A. A. Novotny, E. C. N. Silva, Topological derivatives applied to fluid flow channel design optimization problems, Structural and Multidisciplinary Optimization (to appear) DOI:10.1007/s00158-016-1399-0. doi:10.1007/s00158-016-1399-0.

[11] N. Van Goethem, A. A. Novotny, Crack nucleation sensitivity analysis, Mathematical Methods in the Applied Sciences 33 (16) (2010) 197-1994.

[12] G. Cardone, S. Nazarov, J. Sokołowski, Asymptotic analysis, polarization matrices, and topological derivatives for piezoelectric materials with small voids., SIAM Journal on Control and Optimization 48 (6) (2010) 3925-3961.

[13] G. Frémiot, W. Horn, A. Laurain, M. Rao, J. Sokołowski, On the analysis of boundary value problems in nonsmooth domains, Dissertationes Mathematicae (Rozprawy Matematyczne) 462 (2009) 149.

[14] M. Iguernane, S. Nazarov, J.-R. Roche, J. Sokolowski, K. Szulc, Topological derivatives for semilinear elliptic equations, International Journal of Applied Mathematics and Computer Science 19 (2) (2009) 191-205.

[15] J. Sokołowski, A. Żochowski, Optimality conditions for simultaneous topology and shape optimization, SIAM Journal on Control and Optimization 42 (4) (2003) 1198-1221.

[16] H. Ammari, H. Kang, Reconstruction of small inhomogeneities from boundary measurements, Lectures Notes in Mathematics vol. 1846, Springer-Verlag, Berlin, 2004.

[17] H. Ammari, H. Kang, Polarization and moment tensors with applications to inverse problems and effective medium theory, Applied Mathematical Sciences vol. 162, Springer-Verlag, New York, 2007.

[18] S. M. Giusti, A. A. Novotny, Topological derivative for an anisotropic and heterogeneous heat diffusion problem, Mechanical Research Communication 46 (2012) 26-33.

[19] S. M. Giusti, L. M. A. Mello, E. C. N. Silva, Piezoresistive device optimization using topological derivative concepts, Structural and Multidisciplinary Optimization 50 (2014) 453-464.

[20] S. M. Giusti, A. A. Novotny, J. Sokołowski, Topological derivative for steady-state orthotropic heat diffusion problem, Structural and Multidisciplinary Optimization 40 (1) (2010) 53-64.

[21] E. Beretta, E. Bonnetier, E. Francini, A. L. Mazzucato, Small volume asymptotics for anisotropic elastic inclusions, Inverse Problems and Imaging 6 (1) (2012) 1-23.

[22] S. A. Nazarov, J. Sokołowski, M. Specovius-Neugebauer, Polarization matrices in anisotropic heterogeneous elasticity, Asymptotic Analysis 68 (4) (2010) 189-221.

[23] M. Bonnet, G. Delgado, The topological derivative in anisotropic elasticity, The Quarterly Journal of Mechanics and Applied Mathematics 66 (4) (2013) 557-586.

[24] V. A. Kozlov, V. G. Maz'ya, A. B. Movchan, Asymptotic analysis of fields in multi-structures, Clarendon Press, Oxford, 1999.

[25] S. G. Lekhnitskii, Theory of Elasticity of an Anisotropic Elastic Body, Holden-Day Series in Mathematical Physics, Holden-Day, Inc., San Francisco, California, 1963.

[26] S. G. Lekhnitskii, S. W. Tsai, T. Cheron, Anisotropic Plates, Gordon and Breach Science Publishers, New York, New York, U.S.A., 1968.

[27] S. Amstutz, H. Andrä, A new algorithm for topology optimization using a level-set method, Journal of Computational Physics 216 (2) (2006) 573-588.

[28] D. E. Campeão, S. M. Giusti, A. A. Novotny, Topology design of plates consedering different volume control methods, Engineering Computations 31 (5) (2014) 826-842.

[29] C. G. Lopes, R. B. dos Santos, A. A. Novotny, Topological derivative-based topology optimization of structures subject to multiple load-cases, Latin American Journal of Solids and Structures 12 (2015) 834-860.

[30] S. Amstutz, Analysis of a level set method for topology optimization, Optimization Methods and Software 26 (4-5) (2011) 555-573.

[31] E. Sanchez-Palencia, Non-homogeneous media and vibration theory, Vol. 127 of Lecture Notes in Physics, Springer-Verlag, Berlin, 1980.

[32] M. H. Saad, Elasticity: Theory, Applications, and Numerics, Academic Press., Oxford, 2005.

[33] J. D. Eshelby, The determination of the elastic field of an ellipsoidal inclusion, and related problems, Proceedings of the Royal Society: Section A 241 (1957) 376-396.

[34] J. D. Eshelby, The elastic field outside an ellipsoidal inclusion, and related problems, Proceedings of the Royal Society: Section A 252 (1959) $561-569$.

[35] T. Mura, Micromechanics of defects in solids, Kluwer academic publishers, Dordrecht, 1987. 